\title{
Occupational cholangiocarcinoma diagnosed 18 years after the end of exposure to 1,2-dichloropropane and dichloromethane at a printing company: a case report
}

Masahiko Kinoshita ${ }^{1 *}\left(\mathbb{D}\right.$, Yasunori Sato ${ }^{5}$, Hiroko Nebiki ${ }^{2}$, Yutaka Tamamori ${ }^{3}$, Naomi Ishii ${ }^{4}$, Takeshi Inoue ${ }^{4}$, Genya Hamano ${ }^{1}$, Akishige Kanazawa ${ }^{1}$ and Shoji Kubo ${ }^{6}$

\begin{abstract}
Background: Cholangiocarcinoma due to exposure to 1.2-dichloropropane (DCP) or dichloromethane (DCM) is classified as occupational cholangiocarcinoma. We report a case of occupational cholangiocarcinoma diagnosed 18 years after the end of exposure to organic solvents at a printing company.

Case presentation: A 41-year-old man presented to our hospital with jaundice and anorexia. He had previously worked for 6 years at a printing company where an outbreak of occupational cholangiocarcinoma occurred and was exposed to high concentrations of organic solvents during his employment. Computed tomography demonstrated lower bile duct obstruction by the bulky nodal metastasis at the hepatoduodenal ligament with upstream biliary dilatation, an intraductal papillary tumor in the dilated left superior lateral bile duct (B2), and enlargement of the periaortic nodes. Clinical diagnosis of an unresectable invasive intraductal papillary neoplasm of the bile duct (IPNB) with extensive nodal metastasis was made. Although chemotherapy and laparoscopic gastrojejunostomy were performed for the duodenal obstruction, the patient died due to rupture of the tumor. Pathological examination of the autopsy specimen revealed well-differentiated adenocarcinoma at the stromal site along Glisson's sheath in segment 3, an IPNB lesion without invasion in B2, and biliary intraepithelial neoplasia and chronic bile duct injury at various sites in the large bile ducts. The bulky lymph node (poorly differentiated adenocarcinoma with partial squamous cell differentiation) invaded the bile duct and duodenum.
\end{abstract}

Conclusions: We report a case of occupational cholangiocarcinoma that developed 18 years after the end of exposure to DCP and DCM. Long-term follow-up is required to carefully survey development of cholangiocarcinoma in workers with an occupational history of exposure to organic solvents.

Keywords: Occupational cholangiocarcinoma, Intraductal papillary neoplasm of the bile duct, Biliary intraepithelial neoplasia, 1,2-Dichloropropane, Dichloromethane

\footnotetext{
* Correspondence: pikopiko0128@yahoo.co.jp

'Department of Hepato-Biliary-Pancreatic Surgery, Osaka City General

Hospital, 2-13-22 Miyakojima-Hondori, Miyakojima-ku, Osaka 534-0021, Japan

Full list of author information is available at the end of the article
} 


\section{Background}

A cholangiocarcinoma outbreak occurred at a printing company in Osaka, Japan [1-3]. In 2013, the Japanese Ministry of Health, Labour and Welfare initially recognized this type of cholangiocarcinoma as an occupational disease [4]. By December 2018, 42 patients, including 18 at a printing company, were diagnosed with occupational cholangiocarcinoma. In these patients, 1.2-dichloropropane (DCP) and dichloromethane (DCM) played a key role in the development of this type of cholangiocarcinoma [1-4].

Here we report a patient diagnosed with cholangiocarcinoma 18 years after the end of exposure to high concentrations of DCP and DCM while working at a printing company in Osaka.

\section{Case presentation}

A 41-year-old man was admitted to our hospital with obstructive jaundice and anorexia. For up to 18 years before admission, he worked at a printing company where an outbreak of cholangiocarcinoma occurred, and he was exposed to high concentrations of DCP and DCM over the 6 years of his employment. Six months before his admission, elevated serum gamma-glutamyl transpeptidase $(\gamma-\mathrm{GTP})$ activity was detected during a regular medical examination. The patient had a history of heavy alcohol consumption.

Results of the laboratory tests performed at the first admission revealed an elevated serum total bilirubin concentration $(10.7 \mathrm{mg} / \mathrm{dL})$ and elevated activity of aspartate aminotransferase $(76 \mathrm{U} / \mathrm{L})$, alanine aminotransferase $(226 \mathrm{U} / \mathrm{L})$, and $\gamma$-GTP $(319 \mathrm{U} / \mathrm{L})$. Though the serum concentration of carbohydrate antigen 19-9 (CA 19-9) was within the reference range $(2.0 \mathrm{ng} / \mathrm{mL})$, concentrations of the carcinoembryonic antigen and s-pancreas-1 antigen were elevated $(17.9 \mathrm{ng} / \mathrm{mL}$ and $103.7 \mathrm{U} / \mathrm{mL}$, respectively). A dynamic abdominal computed tomography $(\mathrm{CT})$ scan exhibited dilatation of the intrahepatic bile ducts with common bile duct obstruction owing to a tumor that was suspected to be an enlarged lymph node (maximum diameter, $45 \mathrm{~mm}$ ) originating in the hepatoduodenal ligament or peripancreatic region (the bulky lymph node) and invading the common bile duct and pancreatic head (Fig. 1a) as well as enlarged para-aortic lymph nodes (Fig. 1b). Although the intrahepatic bile ducts were entirely dilated, cystic dilatation of the intraductal tumor suspected as an intraductal papillary neoplasm of the bile duct (IPNB) was identified on $\mathrm{CT}$ and magnetic resonance cholangiopancreatography at the proximal side of the biliary branch in
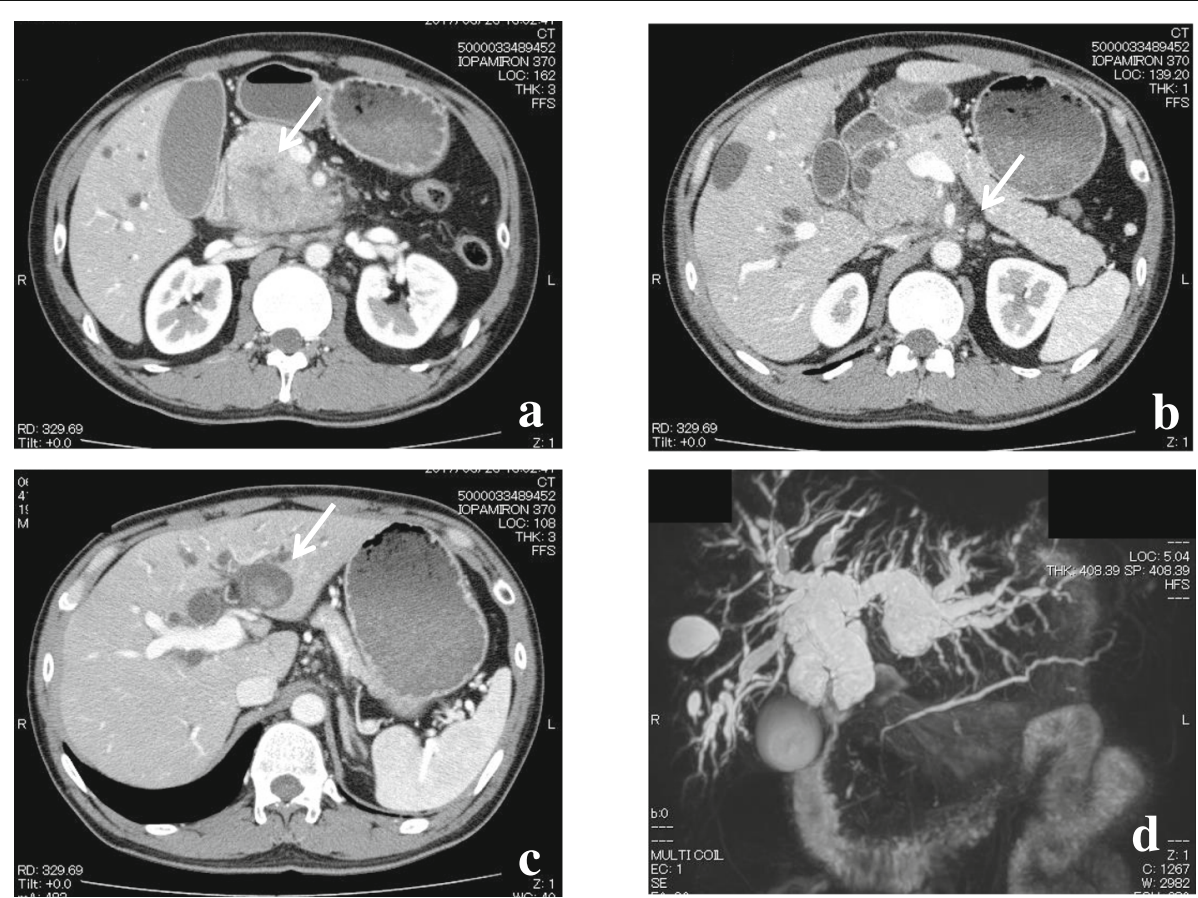

Fig. 1 a Dynamic abdominal computed tomography (CT) revealed a tumor suspected as a lymph node (maximum diameter, 45 mm) around the hepatoduodenal ligament with invasion to the common bile duct and the pancreatic head (arrow). b Para-aortic lymph node enlargement was shown on dynamic CT (arrow). c Cystic dilatation with intraductal tumor, which was suspected as intraductal papillary neoplasm of the bile duct, was identified at the proximal side of the biliary branch in segment 2 (B2) on dynamic CT (arrow). d Dilatation of the intrahepatic bile duct was shown on magnetic resonance cholangiopancreatography 
segment 2 (B2) (Fig. 1c, d). Adenocarcinoma cells were detected on biliary cytology with endoscopic retrograde cholangiopancreatography (ERCP). These findings indicated a cholangiocarcinoma as invasive IPNB with extensive lymph node metastases in the hepatoduodenal ligament and in the para-aortic lesion, with curative surgery considered impossible.

After a metallic stent was inserted at the stenosis of the common bile duct during ERCP, he received chemotherapy with a combination of gemcitabine and cisplatin. After 5 cycles of chemotherapy, the size of the intraductal tumor at B2 remained unchanged; however, the bulky lymph node grew up to $120 \mathrm{~mm}$ in diameter, evidently invading/obstructing the duodenum (Fig. 2a, b). Therefore, we performed laparoscopic gastrojejunostomy with Billroth II reconstruction. Although oral ingestion was achieved after the operation, the patient developed sudden abdominal pain, and an inflammatory response was detected on laboratory test results (white blood cell count of 37,010 cells $/ \mu \mathrm{L}$ and C-reactive protein of $30.9 \mathrm{mg} / \mathrm{dL}$ ) at 19 days after the operation. Abdominal CT revealed ascites and free air around the bulky lymph node (Fig. 2c). During the emergency laparotomy, the bulky lymph node invading the surrounding organs ruptured with a large amount of purulent ascites. The ruptured orifice was covered with the greater omentum, followed by multiple placements of surgical drains. However, the patient's general condition gradually worsened, and he died 8 days after the second operation ( 8 months after admission).

\section{Macroscopic and pathological findings of the autopsy specimen}

Macroscopic findings during the autopsy examination revealed the bulky lymph node invading the duodenum and transverse colon (Fig. 3a). An intraductal tumor was also observed in B2 (Fig. 3b).

Locations of pathological lesions were mapped on the biliary tree, with reference from preoperative radiologic imaging and gross autopsy findings (Fig. 4). We histologically defined carcinoma, biliary intraepithelial neoplasia (BilIN), IPNB, and chronic bile duct injury according to the World Health Organization classification for intrahepatic cholangiocarcinoma [5]. Pathological examination of the autopsy specimens with H\&E staining revealed that the bulky lymph node was diagnosed as a poorly differentiated adenocarcinoma with partial squamous epithelial differentiation (Fig. 4b, c) and invasion to the duodenum, common bile duct, pancreas, and liver. BilIN (Fig. 4d, g) and chronic bile duct injury (Fig. 4a) were identified at various sites in the large bile ducts, and intermediategrade IPNB without invasion was seen in B2 (Fig. 4h, i). In addition, a well-differentiated tubular adenocarcinoma, in
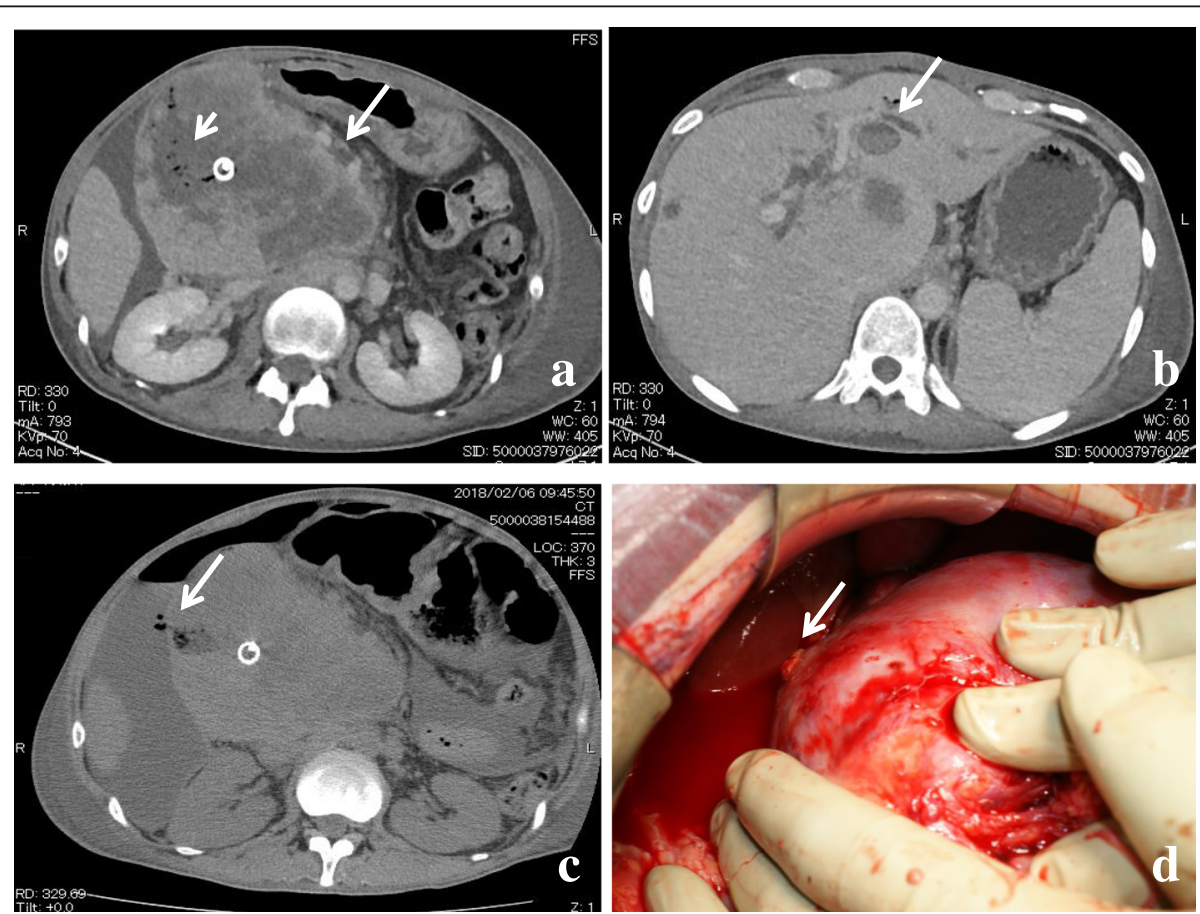

Fig. 2 a Dynamic CT after 5 cycles of chemotherapy. Lymph node metastasis spread (maximum diameter, 120 mm) (arrow). The duodenum was strongly depressed, and air was observed in the tumor (short arrow). $\mathbf{b}$ The size of the intraductal tumor at B2 did not change after the patient received chemotherapy (arrow). c Abdominal CT revealed ascites and continuous free air inside the abdominal tumor as lymph node metastasis in the hepatoduodenal ligament (arrow). $\mathbf{d}$ Intraoperative macroscopic findings. The ruptured tumor was observed in the hepatoduodenal ligament (arrow) 

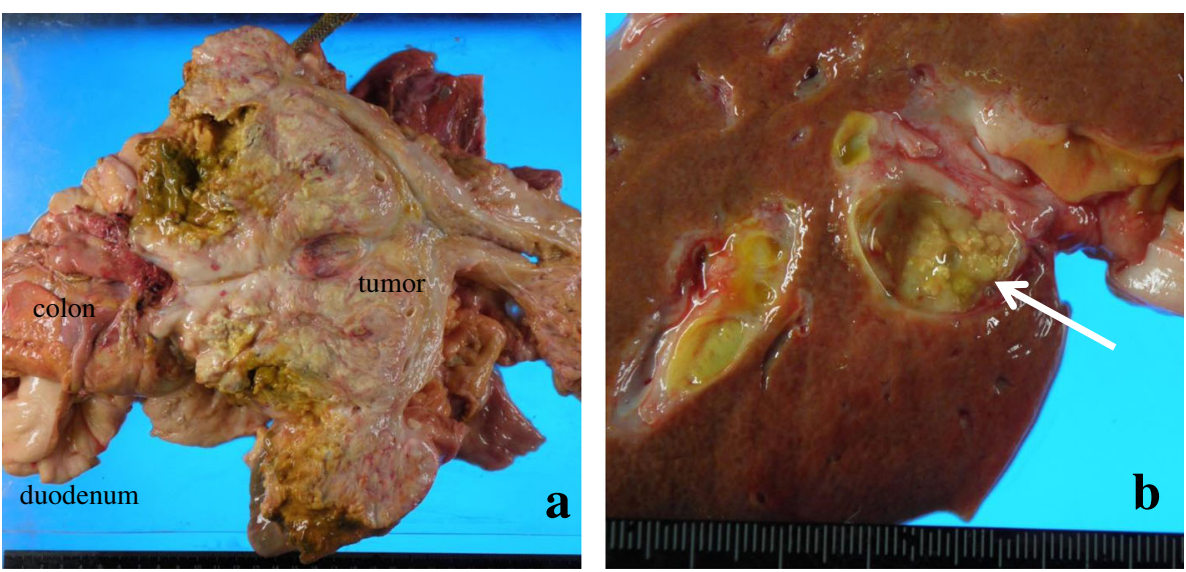

Fig. 3 Macroscopic findings of the large tumor in the hepatoduodenal ligament and liver. a Large tumor with invasion to the duodenum and transverse colon. $\mathbf{b}$ Intraductal tumor (arrow) at the proximal side of the bile ducts in segment 2

which the histologic type differed from enlarged lymph node metastasis, was detected at the stromal site of Glisson's sheath around the proximal side of the bile duct in segment 3 (B3) (Fig. 4e, f). Although BilIN was detected in the large bile ducts around this stromal adenocarcinoma lesion (Fig. 4d), an invasive carcinoma was not obvious in the biliary epithelium of B3, and adenocarcinoma was not detected in any other organs.

Immunological staining using primary antibodies against $\gamma \mathrm{H} 2 \mathrm{AX}$ (1:100 Rabbit Monoclonal; Novus Biologicals, Littleton, CO, USA), which is a marker for double-strand DNA injuries; S100P (1:100 Rabbit Monoclonal, Epitomics), which is a marker for malignant transformation;

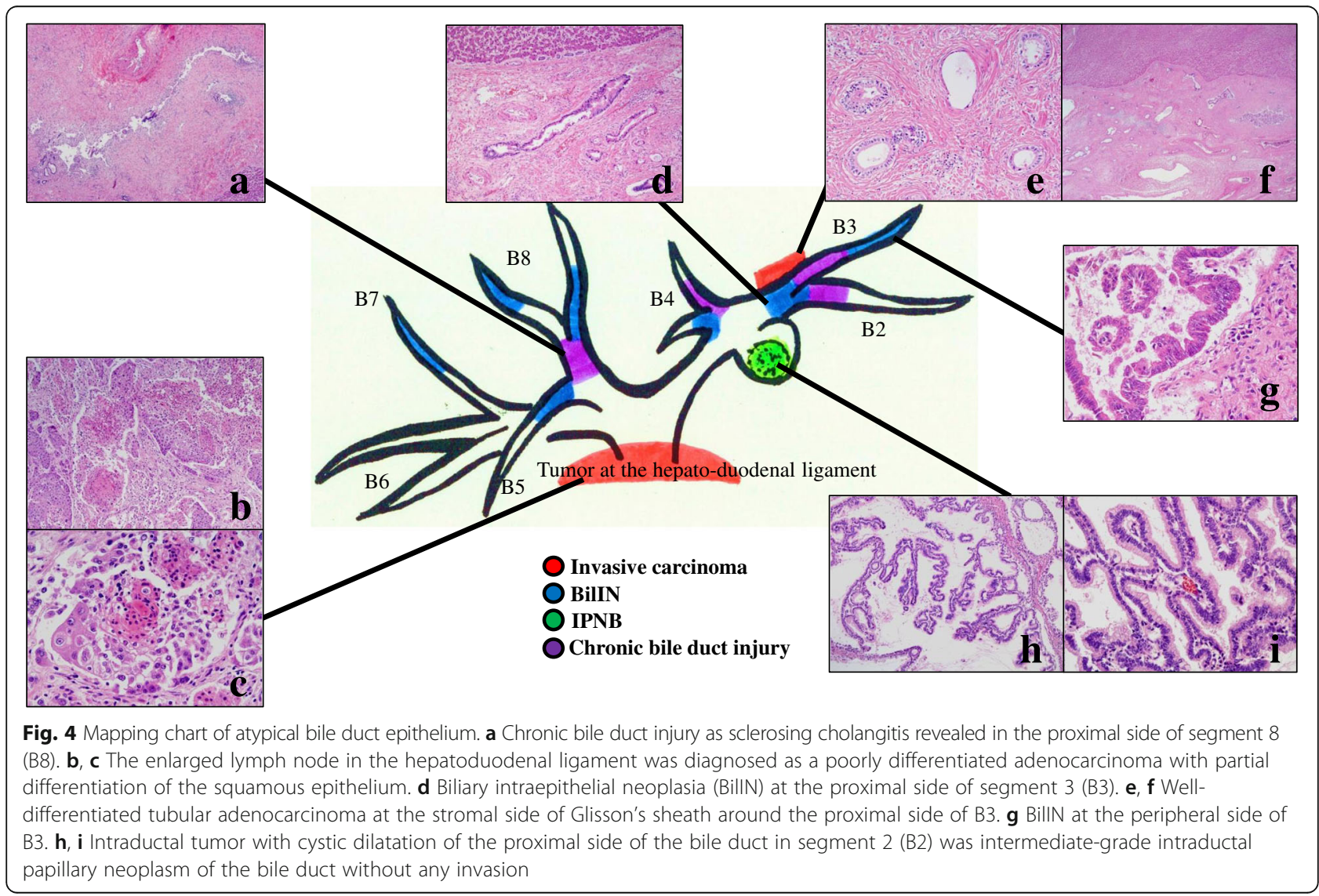


and primary antibodies against PD-L1 (clone 28-8, 1:500; Abcam) were performed. Almost all portions of the invasive carcinoma, BilIN, and IPNB had positive expressions of $\gamma \mathrm{H} 2 \mathrm{AX}$ and S100P. Although $\gamma \mathrm{H} 2 \mathrm{AX}$ expression was also identified within the non-neoplastic biliary epithelium, S100P expression was absent or relatively weak (Table 1, Fig. 5a-j). Neither $\gamma \mathrm{H} 2 \mathrm{AX}$ nor S100P expression was detected in hepatocytes. PD-L1 expression was absent in tumor cells at the stromal side of segment 3 of Glisson's sheath and non-neoplastic epithelium (Table 1, Fig. 5l, o). Although it was $<5 \%$, positive PD-L1 expression was detected in the cells of the bulky lymph node, BilIN, and IPNB (Table 1, Fig. 5k, m, n).

\section{Discussion}

According to our previous studies, elevated serum $\gamma$-GTP activity, regional dilatation of the bile duct without tumor-induced obstruction and biliary findings similar to those of primary sclerosing cholangitis [6] on diagnostic imaging, presence of precancerous or early cancerous lesions, and nonspecific bile duct injuries are characteristics of patients with occupational cholangiocarcinoma [1, 3, 7-9]. Moreover, multicentric and multistep carcinogenesis with a high frequency of somatic mutations via chronic bile duct injury and pre- or early cancerous lesions have also been suggested $[7,10]$. In our case, pathological findings indicated injury at various sites of the large bile ducts, with pre- or early malignant lesions such as IPNB and BilIN developing at various sites. Although primary cholangiocarcinoma was not obvious in the large bile ducts of the autopsy specimen, it could develop at the common bile duct and form a large mass with lymph node metastasis with invasion to the surrounding organs and tissues. Dilatation of the intrahepatic bile ducts without tumor-induced obstruction was not apparent in this case because the intrahepatic bile ducts were dilated owing to the obstructed common bile duct; however, other characteristics, such as a wide range of precancerous lesion, were confirmed. Occupational cholangiocarcinoma is certified by application to the Ministry of Health, Labour and Welfare. The patient did not apply as to have occupational cholangiocarcinoma owing to his will and lack of a legally authorized representative. However, this patient was assumed to have "occupational cholangiocarcinoma" due to his history of exposure to high concentrations of
DCP and DCM for 6 years at the printing company where he worked, in addition to typical clinicopathological findings of occupational cholangiocarcinoma.

The previous study reported that the longest period between the end of the exposure to such organic solvents and a diagnosis of occupational cholangiocarcinoma was 9 years and 7 months [1]. In the present case, cholangiocarcinoma was diagnosed 18 years after the end of exposure, and cholangiocarcinoma in addition to pre- or early cancerous lesions and chronic bile duct injury were observed. Moreover, immunopathological examination also revealed that $\gamma \mathrm{H} 2 \mathrm{AX}$ expression, which is a marker of double-strand DNA injuries, was detected at various sites of the large bile ducts, including the non-neoplastic bile ducts; S100P expression was detected in cholangiocarcinoma as well as premalignant or early malignant lesions, such as BilIN and IPNB, as previously reported [7]. These findings suggest that past exposure of organic solvents insidiously induces multiple and sporadic lesions with a wide range of histological atypia and continuous DNA injury in spite of the long duration after the end of exposure. Therefore, for workers who are exposed to such organic solvents, a follow-up to monitor for cholangiocarcinoma because the potential for malignant transformation persists in the long-term is necessary, even after years after the end of exposure.

In the present case, cholangiocarcinoma was diagnosed at an advanced stage. Although elevated serum $\gamma$-GTP activity was detected at the patient's medical examination, further examinations were not performed. We reported that abnormal liver function serum, especially $\gamma$-GTP activity, and elevated tumor markers were frequently seen in patients with occupational cholangiocarcinoma [1-3], and measurement of liver function tests and tumor markers is important in detecting occupational cholangiocarcinoma [11]. A regular examination to detect cholangiocarcinoma is necessary in workers with a history of exposure to such organic solvents.

In patients with occupational cholangiocarcinoma, the efficacy of chemotherapy remains unclear [12]. In our patient, positive PD-L1 expression was detected in the cells of the large tumor around the hepatoduodenal ligament. We reported on the hypermutation and positive PD-L1 expression of occupational cholangiocarcinoma $[10,13]$. Hence, we believe that anti-PD-1 antibody

Table 1 Results of immunohistochemical analysis for autopsy specimen

\begin{tabular}{llllll}
\hline & Tumor around the hepatoduodenal ligament & Adenocarcinoma at the stromal site of B3 & IPNB & BillN & Non-neoplastic bile duct \\
\hline $\mathrm{Y}-\mathrm{H} 2 \mathrm{AX}$ & + & + & ++ & ++ & ++ \\
$\mathrm{S} 100 \mathrm{P}$ & ++ & ++ & ++ & ++ & - \\
$\mathrm{PD}-\mathrm{L} 1$ & $+(<5 \%)$ & - & $+(<5 \%)$ & $+(<5 \%)$ & - \\
\hline
\end{tabular}

+ : partially positive; ++: diffusely positive; - : negative

IPNB intraductal papillary neoplasm of the bile duct, BillN biliary intraepithelial neoplasia 


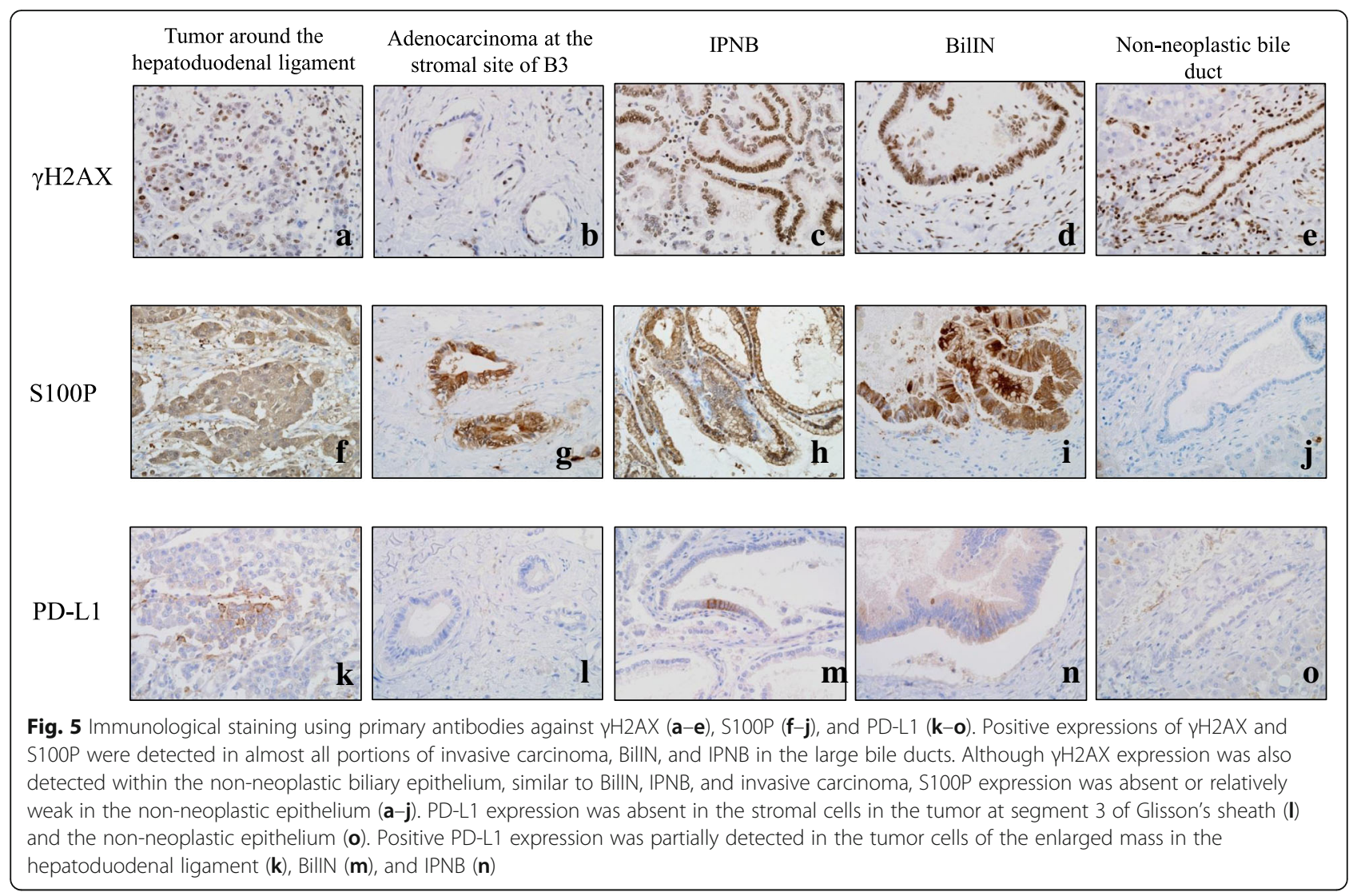

therapy is a feasible alternative therapy for occupational cholangiocarcinoma.

In conclusion, workers with a history of exposure to high concentrations of DCP and/or DCM require longterm follow-up to monitor for the development of cholangiocarcinoma after the end of exposure because the potential for malignant transformation persists over the long term.

\section{Abbreviations}

BillN: Biliary intraepithelial neoplasia; $\mathrm{CT}$ : Computed tomography; DCM: Dichloromethane; DCP: 1.2-Dichloropropane; ERCP: Endoscopic retrograde cholangiopancreatography; IPNB: Intraductal papillary neoplasm of the bile duct; MRCP: Magnetic resonance cholangiopancreatography; PD1: Programmed death-1; PD-L1: Programmed death-1 ligand; үGTP: Gammaglutamyl transpeptidase

\section{Acknowledgements}

The authors would like to thank Enago (www.enago.jp) for the English language review.

\section{Funding}

This work was supported in part by the Japan Society for the Promotion of Science KAKENHI Grant Number 26462048 (Elucidation of carcinogenesis and establishment of treatment strategy for intrahepatic cholangiocarcinoma by genetic and immunological analyses).

\section{Availability of data and materials}

Sharing of data is not applicable because no datasets were generated or analyzed during the current study. Please contact the author for data requests.

\section{Authors' contributions}

MK designed and described the article. HN, YT, GH, and AK advised on the content of the manuscript. SK supervised the editing of the manuscript. YS, $\mathrm{NI}$, and $\mathrm{TI}$ were responsible for the diagnosis based on the pathological findings. All authors read and approved the final manuscript.

\section{Ethics approval and consent to participate}

The institutional ethics committee approved the publication of this case report (No. 1807043).

\section{Consent for publication}

Written informed consent was obtained from the patient for the publication of this case report and the accompanying images.

\section{Competing interests}

The authors declare that they have no competing interests.

\section{Publisher's Note}

Springer Nature remains neutral with regard to jurisdictional claims in published maps and institutional affiliations.

\section{Author details}

'Department of Hepato-Biliary-Pancreatic Surgery, Osaka City General Hospital, 2-13-22 Miyakojima-Hondori, Miyakojima-ku, Osaka 534-0021, Japan. ${ }^{2}$ Department of Gastroenterology, Osaka City General Hospital, 2-13-22 Miyakojima-hondori, Miyakojima-ku, Osaka 534-0021, Japan. ${ }^{3}$ Department of Gastroenterological Surgery, Osaka City General Hospital, 2-13-22 Miyakojima-hondori, Miyakojima-ku, Osaka 534-0021, Japan. ${ }^{4}$ Department of Pathology, Osaka City General Hospital, 2-13-22 Miyakojima-hondori, Miyakojima-ku, Osaka 534-0021, Japan. ${ }^{5}$ Department of Human Pathology, Kanazawa University Graduate School of Medicine, 13-1 Takaramachi, Kanazawa 920-8640, Japan. ${ }^{6}$ Department of Hepato-Biliary-Pancreatic Surgery, 
Osaka City University Graduate School of Medicine, 1-4-3 Asahimachi, Abenoku, Osaka 545-8585, Japan.

Received: 28 February 2019 Accepted: 5 April 2019

Published online: 23 April 2019

\section{References}

1. Kubo S, Nakanuma Y, Takemura S, Sakata C, Urata Y, Nozawa A, et al. Case series of 17 patients with cholangiocarcinoma among young adult workers of a printing company in Japan. J Hepatobiliary Pancreat Sci. 2014;21:479-88.

2. Kumagai S, Kurumatani N, Arimoto A, Ichihara G. Cholangiocarcinoma among offset colour proof-printing workers exposed to 1,2-dichloropropane and/or dichloromethane. Occup Environ Med. 2013;70:508-10.

3. Kubo S, Kinoshita M, Takemura S, Tanaka S, Shinkawa H, Nishioka T, et al. Characteristics of printing company workers newly diagnosed with occupational cholangiocarcinoma. J Hepatobiliary Pancreat Sci. 2014;21:809-17.

4. Ministry of Health, Labour and Welfare. Occupational biliary tract cancer cases in Japan. 2013. http://www.mhlw.go.jp/english/policy/employ-labour/ labour-standards/Occupational.html.

5. Nakanuma Y, Curado MP, Franceschi S, Gores G, Paradis V, Sripa B, et al. Intrahepatic cholangiocarcinoma. In: Bosman FT, Carnoiro F, Hruba RH, Theise ND, editors. WHO classification of tumors of the digestive system. Lyon: IARC Press; 2010. p. 217-24

6. Koyama K, Kubo S, Ueki A, Shimono T, Takemura S, Tanaka S, et al. MR imaging and MR cholangiopancreatography of cholangiocarcinoma developing in printing company workers. Jpn J Radiol. 2017:35:233-41.

7. Kinoshita M, Kubo S, Nakanuma Y, Sato Y, Takemura S, Tanaka S, et al, Pathological spectrum of bile duct lesions from chronic bile duct injury to invasive cholangiocarcinoma corresponding to bile duct imaging findings of occupational cholangiocarcinoma. J Hepatobiliary Pancreat Sci. 2016;23:92-101.

8. Hamano G, Kubo S, Takemura S, Tanaka S, Shinkawa H, Kinoshita M, et al. Comparison of clinicopathological characteristics between patients with occupational and non-occupational intrahepatic cholangiocarcinoma. J Hepatobiliary Pancreat Sci. 2016;23:389-96.

9. Kubo S, Takemura S, Tanaka S, Shinkawa H, Kinoshita M, Hamano G, et al. Occupational cholangiocarcinoma caused by exposure to 1,2dichloropropane and/or dichloromethane. Ann Gastroenterol Surg. 2018:2:99-105.

10. Mimaki S, Totsuka Y, Suzuki Y, Nakai C, Goto M, Kojima M, et al. Hypermutation and unique mutational signatures of occupational cholangiocarcinoma in printing workers exposed to haloalkanes. Carcinogenesis. 2016;37:817-26.

11. Kubo S, Takemura S, Tanaka S, Nishioka T, Kinoshita M, Hamano G, et al. Screening and surveillance for occupational cholangiocarcinoma in workers exposed to organic solvents. Surg Today. 2016:46:705-12.

12. Kubo S, Takemura S, Tanaka S, Shinkawa H, Kinoshita M, Hamano G, et al. Outcomes after resection of occupational cholangiocarcinoma. J Hepatobiliary Pancreat Sci. 2016;23:556-64.

13. Sato Y, Kinoshita M, Takemura S, Tanaka S, Hamano G, Nakamori S, et al. The PD-1/PD-L1 axis may be aberrantly activated in occupational cholangiocarcinoma. Pathol Int. 2017:67:163-70.

\section{Submit your manuscript to a SpringerOpen ${ }^{\circ}$ journal and benefit from:}

- Convenient online submission

Rigorous peer review

Open access: articles freely available online

High visibility within the field

- Retaining the copyright to your article

Submit your next manuscript at $\boldsymbol{\nabla}$ springeropen.com 